\title{
Diabetes Device Use and Glycemic Control among Youth with Type 1 Diabetes: A Single-Center, Cross-Sectional Study
}

\author{
Khalid Sheikh $\mathbb{D}^{1},{ }^{1}$ Sara K. Bartz, ${ }^{2}$ Sarah K. Lyons $\mathbb{D}^{2},{ }^{2}$ and Daniel J. DeSalvo $\mathbb{D}^{2}$ \\ ${ }^{1}$ University of Houston, Houston, TX, USA \\ ${ }^{2}$ Baylor College of Medicine/Texas Children's Hospital, Houston, TX, USA
}

Correspondence should be addressed to Daniel J. DeSalvo; desalvo@bcm.edu

Received 25 January 2018; Accepted 11 June 2018; Published 29 July 2018

Academic Editor: Marco Songini

Copyright (C) 2018 Khalid Sheikh et al. This is an open access article distributed under the Creative Commons Attribution License, which permits unrestricted use, distribution, and reproduction in any medium, provided the original work is properly cited.

\begin{abstract}
Aim. The purpose of this cross-sectional study was to determine the rates of diabetes device use (insulin pump and continuous glucose monitor (CGM)) and association with glycemic control in youth with type 1 diabetes in a large, diverse pediatric center. Methods. Demographic and clinical data were obtained from 1992 patients who met the eligibility criteria (age $<26$ years, diabetes duration $\geq 1$ year, and $\geq 1$ clinic visit in the preceding 12 months). Statistical analyses assessed the likelihood of device use based on demographic characteristics and the association between device use and glycemic control based on most recent hemoglobin A1c (HbA1c). Results. Mean age was $13.8 \pm 4.2$ years, $50.7 \%$ were female, diabetes duration was $6.2 \pm 4$ years, and mean $\mathrm{HbAlc}$ was $8.7 \pm 1.8 \%$. Overall, $38.2 \%$ of patients were on pump therapy and $18.5 \%$ were on CGM. Patients who were non-Hispanic $(\mathrm{NH})$ white, privately insured, and with primary English-speaking parent(s) had higher rates of insulin pump use, as well as CGM use $(P<0.001$ for both). Female patients had higher rates of pump use only $(P<0.01)$. Private health insurance, $\mathrm{NH}$ white race/ethnicity, and CGM use were each associated with lower $\operatorname{HbAlc}(P=0.03,<0.001$, and $<0.008$, resp.). Conclusion. At a large, diverse, pediatric diabetes center, disparities in diabetes device use were present across sex, race/ethnicity, health insurance coverage, and primary language of parent(s). CGM use was associated with lower HbA1c. Quality improvement efforts are underway to ensure improved access, education, and clinical programs for advanced diabetes devices for T1D patients.
\end{abstract}

\section{Introduction}

Intensive treatment of type 1 diabetes (T1D) leads to improved glycemic control resulting in reduced risk of microvascular complications [1-3]. Current American Diabetes Association (ADA) recommendations for pediatric patients with T1D are aimed at achieving near normal glycemia (HbAlc $<7.5 \%$ for children and $<7.0 \%$ for young adults) while avoiding severe hypoglycemic events [4]; however, the vast majority of youth and young adults with T1D are not meeting glycemic targets. Fewer than $25 \%$ of pediatric patients in the T1D Exchange Registry, a US-based registry comprised of over 26,000 individuals with T1D, achieve the ADA-recommended HbAlc target [5].

Insulin pumps and continuous glucose monitors (CGM) are advanced diabetes management devices that may lead to improved glycemic control compared to traditional insulin injections with self-monitoring of blood glucose (SMBG) [6-11]. Compared to injections, insulin pump therapy offers a more physiologic method of insulin delivery by simulating the normal diurnal pattern of basal insulin secretion in conjunction with prandial or correction boluses [12]. CGM is an emerging technology that provides a continuous measure of interstitial fluid glucose levels to provide real-time trends and alerts to glucose excursions [13]. Despite their potential benefit for improving glycemic control, uptake of these technologies has been limited with $60 \%$ of T1D Exchange Registry participants using an insulin pump and a mere $11 \%$ using CGM [5].

In this study, we evaluated the rates of advanced diabetes device use and association with glycemic control among pediatric and young adult patients with T1D at Texas Children's 
Hospital (TCH) - a large, academic, tertiary urban hospital with a diverse patient population.

\section{Methods}

The study was approved by the Baylor College of Medicine Institutional Review Board. We deployed the EPIC ${ }^{\circledR}$ electronic medical record population health management system for the Texas Children's Hospital diabetes clinic patient registry to generate a comprehensive data report to perform a cross-sectional analysis. Recorded variables included age, sex, race/ethnicity, diabetes type, date of diagnosis, insurance coverage, primary spoken language of parent(s), insulin management category, use of CGM, and most recent $\mathrm{HbA1c}$ measurement. Medical insurance was classified as public for patients with coverage through Medicaid or Children's Health Insurance Program (CHIP). Eligibility criteria included patients $<26$ years of age with a clinical diagnosis of T1D of at least 1-year duration with at least one diabetes clinic visit between July 1, 2015, and June 30, 2016.

2.1. Statistical Analysis. Fisher's exact test was used to compare the distribution of patient characteristics between those who use technology (CGM and/or insulin pump) and those who do not. The multiple logistic regression model including sex, primary language of parent(s), insurance type (public or private), and race/ethnicity was used to estimate the odds of not using pump therapy and not using CGM. A general linear model (least square means) was used to analyze significant associations between the predictor variables and $\mathrm{HbA1c}$. Unadjusted HbA1c data was analyzed by $t$-test for 2-group comparisons and analysis of variance (ANOVA) for multiple-group comparisons. Data analyses were performed using SAS version 9.4 (2011 SAS Institute Inc., Cary, NC). Two-sided $P$ values $<0.05$ were considered statistically significant. Data are presented as mean \pm standard deviation unless otherwise specified.

\section{Results}

A total of 1992 T1D patients met the criteria, with $50.7 \%$ female, mean age of $13.8 \pm 4.2$ years, duration of diabetes of $6.2 \pm 4.0$ years, and $\mathrm{HbA} 1 \mathrm{c}$ of $8.7 \pm 1.8 \%$ (Table 1 ).

Overall, 761 (38.2\%) patients were using an insulin pump and 369 (18.5\%) were using CGM for diabetes management. Females were more likely to use insulin pumps than males $(P<0.01)$, while rates of CGM use were similar between males and females $(P=0.06)$. Non-Hispanic $(\mathrm{NH})$ whites were more likely to use insulin pumps $(P<0.001)$ and CGM $(P<0.001)$ than minority patients. Patients with private health insurance were more likely to use insulin pumps $(P<0.001)$ and CGM $(P<0.001)$ than those with public health insurance or no insurance. Patients with Englishspeaking parent(s) were more likely to use insulin pumps $(P<0.001)$ and CGM $(P<0.001)$ than those with Spanishspeaking parent(s) (Table 2$)$.

In multiple logistic regression modeling, all variables maintained significant associations with pump use after
TABle 1: Demographic characteristics of the 1992 T1D patients.

\begin{tabular}{lc}
\hline & $N(\%)$ \\
\hline Sex & \\
Male & $982(49.3 \%)$ \\
Female & $1010(50.7 \%)$ \\
Age & \\
$2-6$ years & $70(3.5 \%)$ \\
$6-<13$ years & $629(31.6 \%)$ \\
$13-<18$ years & $905(45.4 \%)$ \\
$18-<26$ years & $388(19.5 \%)$ \\
Race/ethnicity & \\
Non-Hispanic white & $1043(52.4 \%)$ \\
Non-Hispanic black & $334(16.8 \%)$ \\
Hispanic & $484(24.3 \%)$ \\
Other & $131(6.6 \%)$ \\
Insurance type & \\
Private & $1325(66.5 \%)$ \\
Public & $639(32.1 \%)$ \\
None & $28(1.4 \%)$ \\
Primary language of parent(s) & \\
English & $1836(92.2 \%)$ \\
Spanish & $156(7.8 \%)$ \\
\hline
\end{tabular}

simultaneously adjusting for the other variables in the model. The odds of not using a pump were greater among males (OR: 1.46; 95\% CI: 1.20, 1.77), patients with Spanishspeaking parent(s) (OR: 2.27; 95\% CI: 1.36, 3.78), and those with public health insurance (OR: 1.97; 95\% CI: 1.54, 2.54). The odds of not using a pump were also greater among Hispanics (OR: 1.82; 95\% CI: 1.37, 2.40) and NH blacks (OR: 5.37; 95\% CI: 3.83, 7.54) than among $\mathrm{NH}$ whites (Figure 1(a)).

Similarly, all variables maintained statistically significant associations with CGM use after simultaneously adjusting for the other variables in the model. The odds of not using CGM were greater among males (OR: 1.32; 95\% CI: 1.03, 1.69), patients with Spanish-speaking parent(s) (OR: 6.59; 95\% CI: $1.51,28.73$ ), and those with public insurance (OR: 11.59; 95\% CI: 6.72, 19.99). The odds of not using CGM were also greater among Hispanics (OR: 1.62; 95\% CI: 1.09, 2.40) and NH blacks (OR: 4.06; 95\% CI: 2.48, 6.66) than among $\mathrm{NH}$ whites (Figure 1(b)).

In the general linear model (least square means) analyzing the association between predictor variables and HbA1c, private health insurance $(P=0.003)$ and $\mathrm{NH}$ whites $(P<0.001)$ were associated with lower HbA1c estimates after simultaneously adjusting for all variables in the model. Neither sex nor primary language of parents was found to have a difference in $\mathrm{HbAlc}$ estimate. CGM use was associated with lower HbA1c estimate $(P<0.008)$, but pump use was not $(P=0.295)$ (Table 3$)$.

In the analysis of unadjusted HbAlc data, publicly insured patients had higher mean $\mathrm{HbAlc}$ than privately 
TABLE 2: Comparison of patient characteristics between those who use diabetes device (pump/CGM) and those who do not.

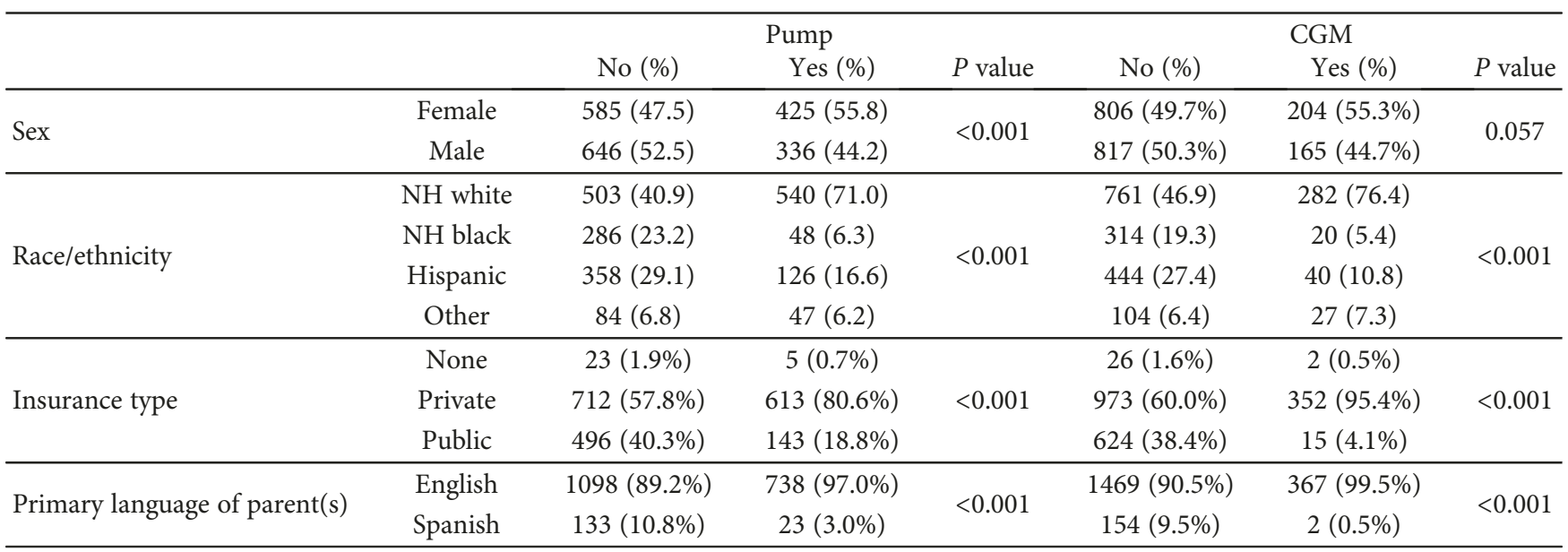

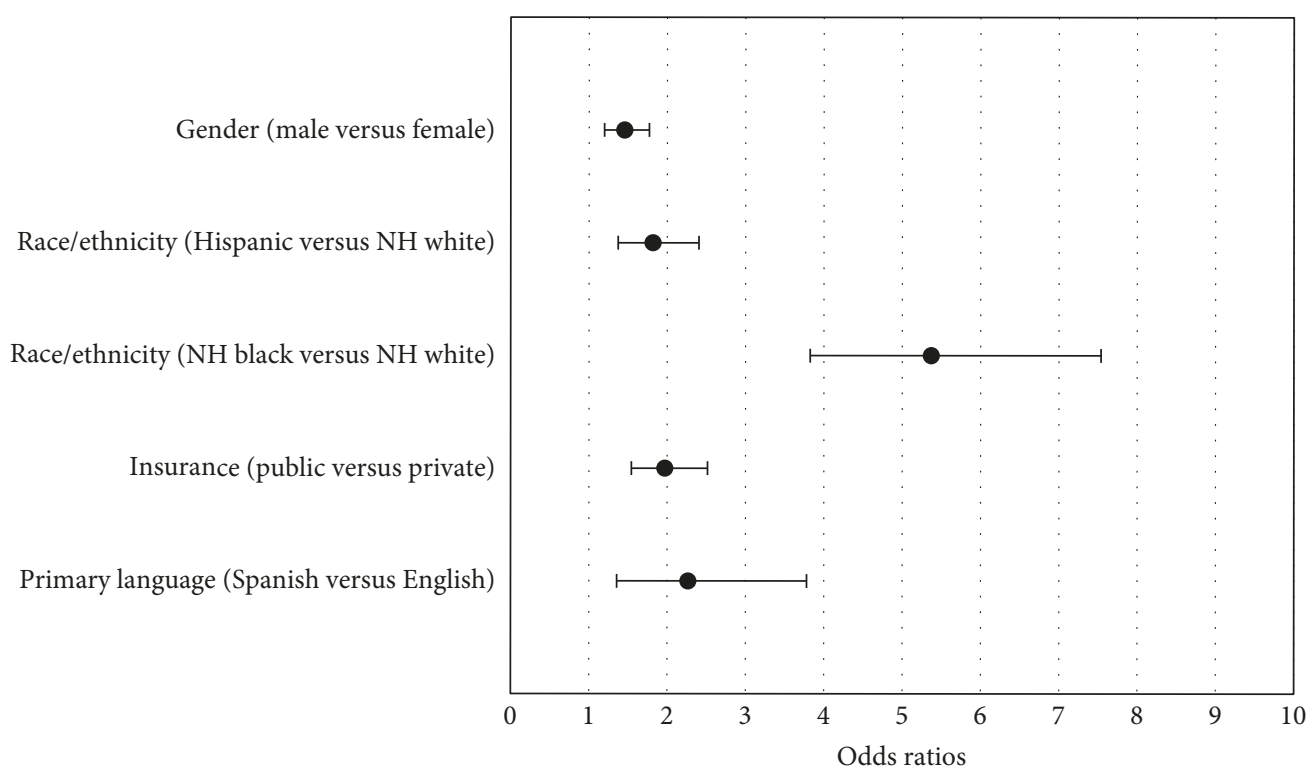

(a)

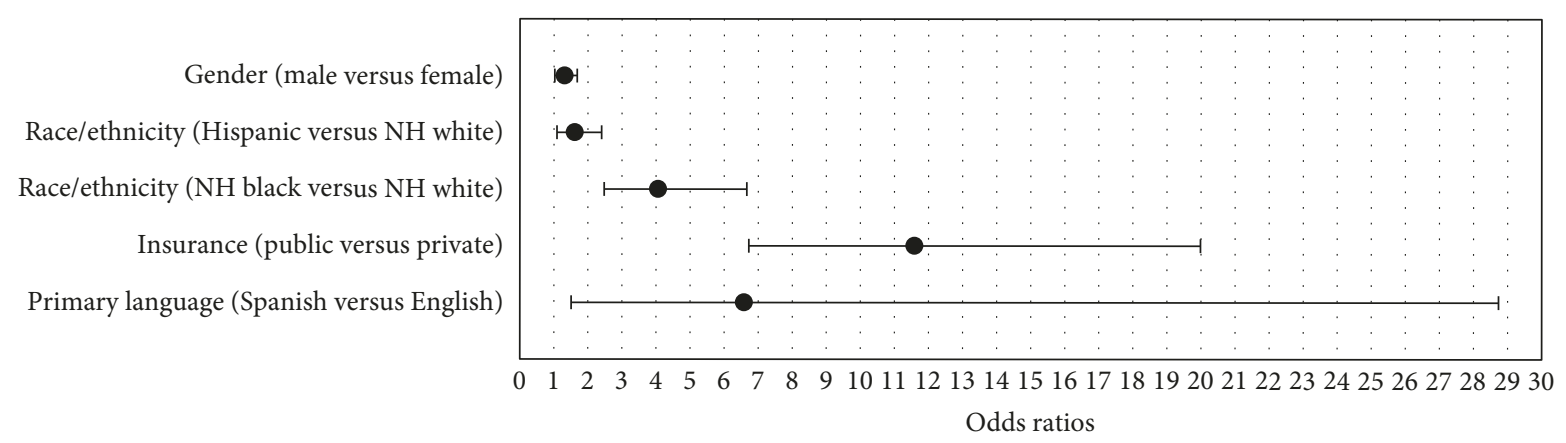

(b)

FIGURE 1: (a) Odds ratios of not using an insulin pump (95\% confidence intervals). (b) Odds ratios of not using CGM (95\% confidence intervals).

insured patients $(9.1 \pm 2.0 \%$ versus $8.4 \pm 1.6 \%, P \leq 0.001)$; however, this difference was not seen between publicly insured pump users and privately insured pump users $(8.5 \pm 1.5 \%$ versus $8.3 \pm 1.4 \%, P=0.279)$ (Figure 2$)$.

\section{Discussion}

This single-center, cross-sectional study analyzed the associations between diabetes device use and demographic 
TABLE 3: Association between patient characteristics and HbAlC.

\begin{tabular}{|c|c|c|c|c|c|c|}
\hline & & HbAlc estimate & Standard error & Lower & Upper & $P$ value \\
\hline \multirow{2}{*}{ Sex } & Female & 8.9 & 0.18 & 8.6 & 9.3 & \multirow{2}{*}{$P=0.525$} \\
\hline & Male & 8.9 & 0.19 & 8.5 & 9.2 & \\
\hline \multirow{4}{*}{ Race/ethnicity } & NH white & 8.5 & 0.19 & 8.2 & 8.9 & \multirow{4}{*}{$P<0.001$} \\
\hline & NH black & 9.6 & 0.20 & 9.3 & 10.0 & \\
\hline & Hispanic & 8.9 & 0.18 & 8.5 & 9.2 & \\
\hline & Other & 8.6 & 0.23 & 8.1 & 9.0 & \\
\hline \multirow{3}{*}{ Insurance type } & None & 8.6 & 0.35 & 7.9 & 9.3 & \multirow{3}{*}{$P=0.003$} \\
\hline & Private & 8.9 & 0.15 & 8.6 & 9.2 & \\
\hline & Public & 9.2 & 0.16 & 8.9 & 9.5 & \\
\hline \multirow{2}{*}{ Primary language } & English & 9.0 & 0.17 & 8.7 & 9.3 & \multirow{2}{*}{$P=0.234$} \\
\hline & Spanish & 8.8 & 0.23 & 8.4 & 9.2 & \\
\hline \multirow{2}{*}{ Pump use } & No & 9.0 & 0.19 & 8.6 & 9.4 & \multirow{2}{*}{$P=0.295$} \\
\hline & Yes & 8.8 & 0.22 & 8.4 & 9.2 & \\
\hline \multirow{2}{*}{ CGM use } & No & 9.1 & 0.18 & 8.7 & 9.4 & \multirow{2}{*}{$P=0.008$} \\
\hline & Yes & 8.7 & 0.21 & 8.3 & 9.1 & \\
\hline
\end{tabular}

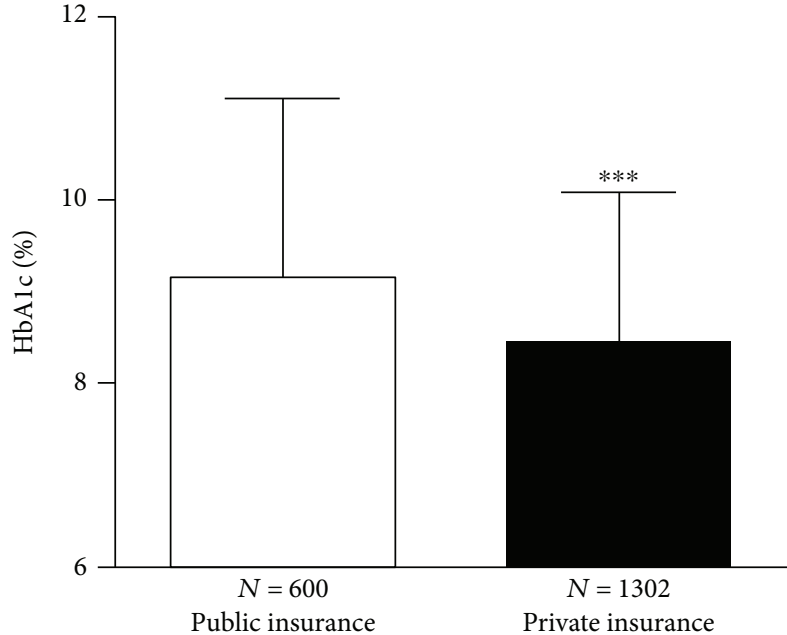

(a)

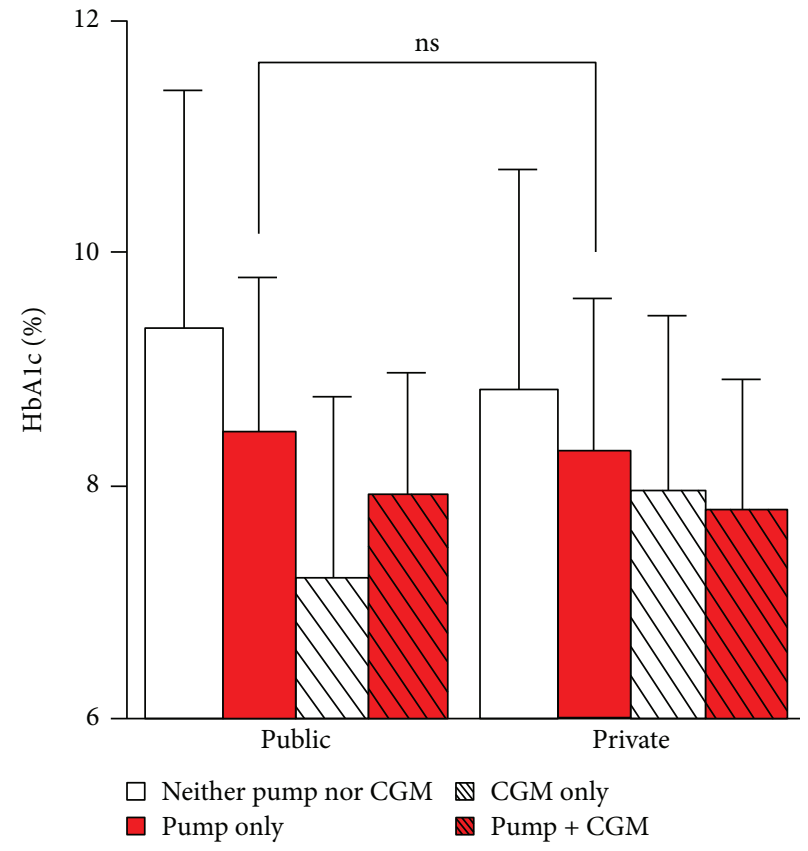

(b)

Figure 2: (a) Unadjusted HbA1c data by insurance type. (b) Unadjusted HbA1c data for insurance type analyzed by diabetes treatment regimen stratified by pump and/or CGM use. $* * *$ indicates $P<0.001$; ns indicates not statistically significant.

factors in pediatric and young adult patients with T1D and between device use and glycemic control. In our large, diverse, pediatric diabetes center, differences in diabetes device use were present for sex, race/ethnicity, health insurance coverage, and primary language of parent(s).

Disparities in device use and glycemic control were found between Hispanic and $\mathrm{NH}$ black patients compared to $\mathrm{NH}$ white patients. These racial/ethnic differences are in line with studies reporting lower rates of device use for Hispanic and
NH black patients compared to NH white patients [14-16]. The previous study suggested that racial/ethnic disparities in diabetes device use may be perpetuated by subconscious racial stereotyping by providers assessing minority patients' preparedness for diabetes devices [16].

Disparities in rates of device use and glycemic control were also present between patients with Spanish-speaking parent(s) compared to those with English-speaking parent(s). The previous study reported language barrier as a 
contributing factor to low diabetes device use, possibly due to inadequate education regarding the benefits of these technologies [17]. Notably, patients who are Hispanic and have Spanish-speaking parents face two potential barriers (minority status and language) to diabetes device uptake. In effort to improve rates of diabetes device use, our diabetes center has recently developed comprehensive education materials, available in both Spanish and English. The clinic also employs bilingual certified diabetes educators proficient in Spanish to deliver the education to Spanish-speaking families.

Previous studies have reported that socioeconomic status impacts diabetes device use due to financial concerns of families $[15,18]$. The difference in rates of diabetes device use between patients with private and public health insurance at our institution was striking. In Texas, public insurance programs including Medicaid and CHIP provide comprehensive coverage for insulin pump therapy but not currently for CGM devices. Therefore, the large disparity in pump use between publicly and privately insured patients was unexpected and suggests that affordability may not be the primary barrier to diabetes device use. The disparities in device use may be due to unmeasured variables such as patient preference or provider biases in interpreting patients' device preparedness. The recent study reported that patients with private insurance have better glycemic control than patients with public insurance [19]. Results from the analysis of our unadjusted data suggest that insulin pump use may mitigate the difference in $\mathrm{HbAlc}$ found between patients on public and private insurance; however, the cross-sectional study design does not determine the causality of pump use in lowering HbAlc.

Our finding that CGM use was associated with improved glycemic control (lower mean HbAlc) is consistent with numerous studies [20-24]. CGM provides patients with a real-time view of the glucose level and trends to augment diabetes treatment decisions on a frequent basis to optimize glycemic control. Additionally, downloading CGM data provides a comprehensive pattern of glycemic trends, allowing providers to make more informed adjustments in insulin regimens [13]. Our findings suggest that CGM use may be more effective in improving glycemic control (lower HbAlc) than insulin pump therapy alone. This suggests that if choosing between one device and the other, CGM may be preferred initially. The introduction of two devices at once may add to the overwhelming feeling in diabetes management, especially in newly diagnosed patients $[24,25]$, so introducing patients to CGM first may lead to improvements in glycemic control while limiting burden of disease on the patient and family. Eventually transitioning patients to sensor-augmented pump therapy may allow patients to benefit from both devices.

Our study has multiple limitations. A major limitation is its cross-sectional design, which does not allow determination of causality of device use with glycemic control. Although we found a correlation between CGM use and lower HbAlc, we were unable to measure the effect of wear time on CGM efficacy. This is a notable limitation since previous studies report that duration of CGM is positively related to reduction in HbAlc $[9,22,26,27]$. An additional limitation is that our analyses did not include parental income or the level of education, which are important factors in diabetes device use [14-16].

Our findings reveal a myriad of potential barriers in utilizing diabetes devices that may assist patients in achieving optimal glycemic control. Many patients face multiple barriers in device uptake (i.e., minority status, insurance coverage, and language), placing them at further disadvantage in initiating pump therapy and/or CGM. Innovative quality improvement efforts, clinical programs, and research interventions must be implemented to overcome potential barriers to diabetes device use and thereby allow more patients to achieve improved glycemic control.

\section{Conclusion}

Differences in rates of diabetes device use were present across sex, race/ethnicity, health insurance coverage, and primary spoken language of parents. The use of CGM was associated with improved glycemic control (lower HbA1c), more so than insulin pump therapy. Strategies to effectively increase and utilize advanced diabetes devices among T1D patients of all race/ethnicities, insurance types, and languages could substantially improve clinical outcomes. To this end, quality improvement efforts are underway at our center to ensure improved access, education, and clinical programs for advanced diabetes devices for T1D patients.

\section{Data Availability}

The authors agree to make our deidentified dataset available by request, immediately upon publication.

\section{Conflicts of Interest}

The authors declare that the funding did not lead to any conflict of interest regarding the publication of this manuscript. Dr. Daniel J. DeSalvo has received honorariums as an advisor to Dexcom (CGM company) and Insulet (maker of the Omnipod insulin pump); however, neither of these products is mentioned or endorsed in the manuscript.

\section{Acknowledgments}

The authors wish to thank the Baylor College of Medicine Institute for Clinical and Translational Research for providing statistical support for this study. Khalid Sheikh received research funding from the Houston Scholars Program at the University of Houston, which was supported by the Provost's Office and the Honors College. The Section of Pediatric Diabetes \& Endocrinology at Baylor College of Medicine/ Texas Children's Hospital provided funding for the bistatistical support for this study.

\section{References}

[1] D. M. Nathan, S. Genuth, J. Lachin et al., "The effect of intensive treatment of diabetes on the development and progression of long-term complications in insulin-dependent diabetes mellitus," The New England Journal of Medicine, vol. 329, no. 14, pp. 977-986, 1993. 
[2] D. M. Nathan, P. A. Cleary, J. Y. Backlund et al., "Intensive diabetes treatment and cardiovascular disease in patients with type 1 diabetes," The New England Journal of Medicine, vol. 353, no. 25, pp. 2643-2653, 2005.

[3] B. Fullerton, K. Jeitler, M. Seitz, K. Horvath, A. Berghold, and A. Siebenhofer, "Intensive glucose control versus conventional glucose control for type 1 diabetes mellitus," Cochrane Database of Systematic Reviews, no. 2, article CD009122, 2014.

[4] American Diabetes Association, "6. Glycemic targets: standards of medical care in diabetes-2018," Diabetes Care, vol. 41, Supplement 1, pp. S55-S64, 2018.

[5] K. M. Miller, N. C. Foster, R. W. Beck et al., "Current state of type 1 diabetes treatment in the U.S.: updated data from the T1D exchange clinic registry," Diabetes Care, vol. 38, no. 6, pp. 971-978, 2015.

[6] S. R. Johnson, M. N. Cooper, T. W. Jones, and E. A. Davis, "Long-term outcome of insulin pump therapy in children with type 1 diabetes assessed in a large population-based casecontrol study," Diabetologia, vol. 56, no. 11, pp. 2392-2400, 2013.

[7] J. C. Pickup and A. J. Sutton, "Severe hypoglycaemia and glycaemic control in type 1 diabetes: meta-analysis of multiple daily insulin injections compared with continuous subcutaneous insulin infusion," Diabetic Medicine, vol. 25, no. 7, pp. 765-774, 2008.

[8] R. W. Beck, I. B. Hirsch, L. Laffel et al., "The effect of continuous glucose monitoring in well-controlled type 1 diabetes," Diabetes Care, vol. 32, pp. 1378-1383, 2009.

[9] D. Deiss, J. Bolinder, J. P. Riveline et al., "Improved glycemic control in poorly controlled patients with type 1 diabetes using real-time continuous glucose monitoring," Diabetes Care, vol. 29, no. 12, pp. 2730-2732, 2006.

[10] M. Rachmiel, Z. Landau, M. Boaz et al., "The use of continuous glucose monitoring systems in a pediatric population with type 1 diabetes mellitus in real-life settings: the AWeSoMe study group experience," Acta Diabetologica, vol. 52, no. 2, pp. 323-329, 2015.

[11] B. Karges, A. Schwandt, B. Heidtmann et al., "Association of insulin pump therapy vs insulin injection therapy with severe hypoglycemia, ketoacidosis, and glycemic control among children, adolescents, and young adults with type 1 diabetes," JAMA, vol. 318, no. 14, pp. 1358-1366, 2017.

[12] J. C. Pickup, "Insulin-pump therapy for type 1 diabetes mellitus," The New England Journal of Medicine, vol. 366, no. 17, pp. 1616-1624, 2012.

[13] D. DeSalvo and B. Buckingham, "Continuous glucose monitoring: current use and future directions," Current Diabetes Reports, vol. 13, no. 5, pp. 657-662, 2013.

[14] S. M. Willi, K. M. Miller, L. A. DiMeglio et al., "Racial-ethnic disparities in management and outcomes among children with type 1 diabetes," Pediatrics, vol. 135, no. 3, pp. 424-434, 2015.

[15] C. A. Paris, G. Imperatore, G. Klingensmith et al., "Predictors of insulin regimens and impact on outcomes in youth with type 1 diabetes: the SEARCH for Diabetes in Youth study," The Journal of Pediatrics, vol. 155, no. 2, pp. 183-189.e1, 2009, e181.

[16] J. M. Valenzuela, A. M. La Greca, O. Hsin, C. Taylor, and A. M. Delamater, "Prescribed regimen intensity in diverse youth with type 1 diabetes: role of family and provider perceptions," Pediatric Diabetes, vol. 12, no. 8, pp. 696-703, 2011.
[17] S. Nam, C. Chesla, N. A. Stotts, L. Kroon, and S. L. Janson, "Barriers to diabetes management: patient and provider factors," Diabetes Research and Clinical Practice, vol. 93, no. 1, pp. 1-9, 2011.

[18] P. V. Commissariat, C. T. Boyle, K. M. Miller et al., "Insulin pump use in young children with type 1 diabetes: sociodemographic factors and parent-reported barriers," Diabetes Technology \& Therapeutics, vol. 19, no. 6, pp. 363-369, 2017.

[19] M. A. Clements, N. C. Foster, D. M. Maahs et al., "Hemoglobin A1c (HbA1c) changes over time among adolescent and young adult participants in the T1D exchange clinic registry," Pediatric Diabetes, vol. 17, no. 5, pp. 327-336, 2016.

[20] D. C. Klonoff, D. Ahn, and A. Drincic, "Continuous glucose monitoring: a review of the technology and clinical use," Diabetes Research and Clinical Practice, vol. 133, pp. 178-192, 2017.

[21] D. Ahn, J. Pettus, and S. Edelman, "Unblinded CGM should replace blinded CGM in the clinical management of diabetes," Journal of Diabetes Science and Technology, vol. 10, no. 3, pp. 793-798, 2016.

[22] Juvenile Diabetes Research Foundation Continuous Glucose Monitoring Study Group, "Effectiveness of continuous glucose monitoring in a clinical care environment: evidence from the Juvenile Diabetes Research Foundation continuous glucose monitoring (JDRF-CGM) trial," Diabetes Care, vol. 33, no. 1, pp. 17-22, 2010.

[23] J. C. Pickup, S. C. Freeman, and A. J. Sutton, "Glycaemic control in type 1 diabetes during real time continuous glucose monitoring compared with self monitoring of blood glucose: meta-analysis of randomised controlled trials using individual patient data," BMJ, vol. 343, no. jul07 1, p. d3805, 2011.

[24] M. Langendam, Y. M. Luijf, L. Hooft, J. H. Devries, A. H. Mudde, and R. J. Scholten, "Continuous glucose monitoring systems for type 1 diabetes mellitus," Cochrane Database of Systematic Reviews, vol. 1, 2012.

[25] J. L. Chiang, M. S. Kirkman, L. M. Laffel, A. L. Peters, and Type 1 Diabetes Sourcebook Authors, "Type 1 diabetes through the life span: a position statement of the American Diabetes Association," Diabetes Care, vol. 37, no. 7, pp. 2034-2054, 2014.

[26] W. V. Tamborlane, R. W. Beck, B. W. Bode et al., "Continuous glucose monitoring and intensive treatment of type 1 diabetes," The New England Journal of Medicine, vol. 359, no. 14, pp. 1464-1476, 2008.

[27] D. Raccah, V. Sulmont, Y. Reznik et al., "Incremental value of continuous glucose monitoring when starting pump therapy in patients with poorly controlled type 1 diabetes: the RealTrend study," Diabetes Care, vol. 32, no. 12, pp. 2245-2250, 2009. 


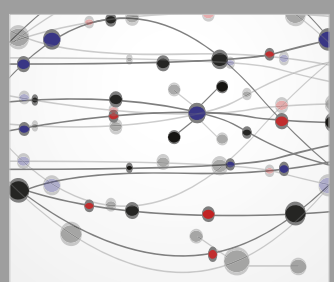

The Scientific World Journal
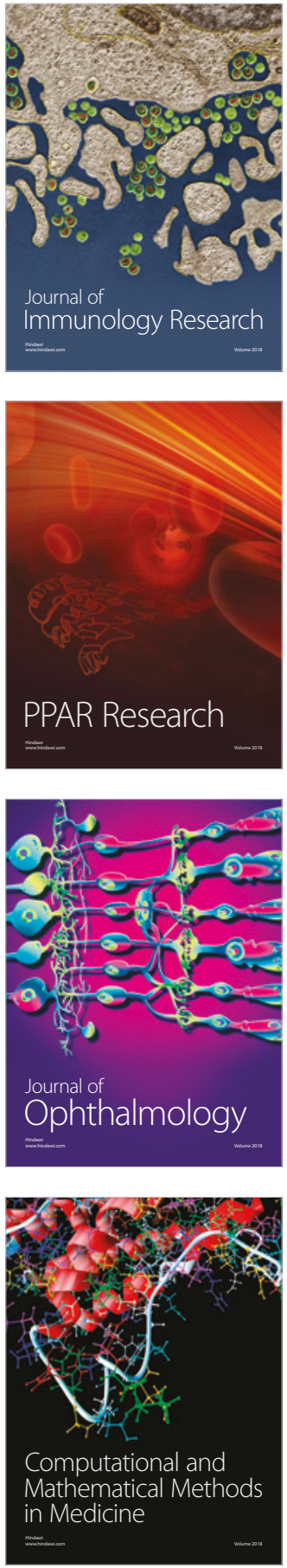

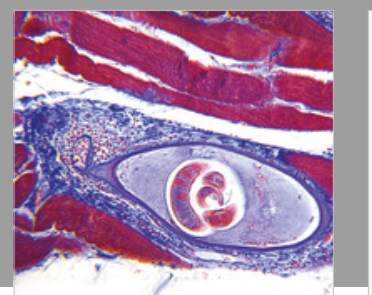

Gastroenterology Research and Practice

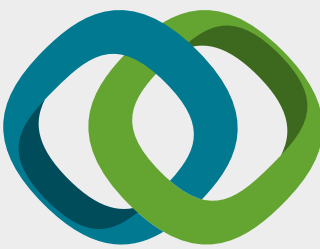

\section{Hindawi}

Submit your manuscripts at

www.hindawi.com
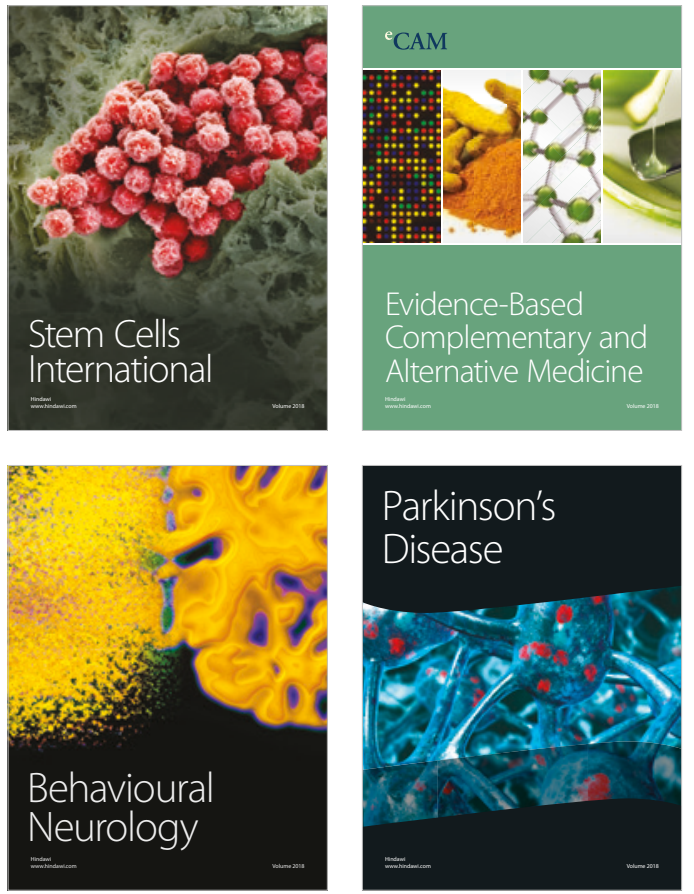

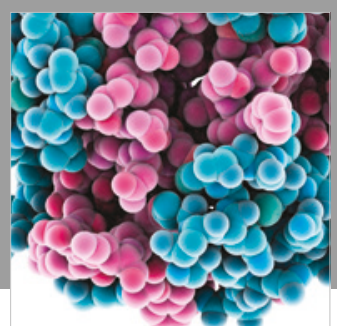

ournal of

Diabetes Research

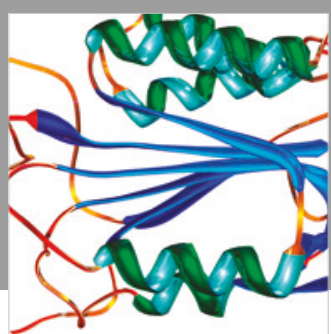

Disease Markers
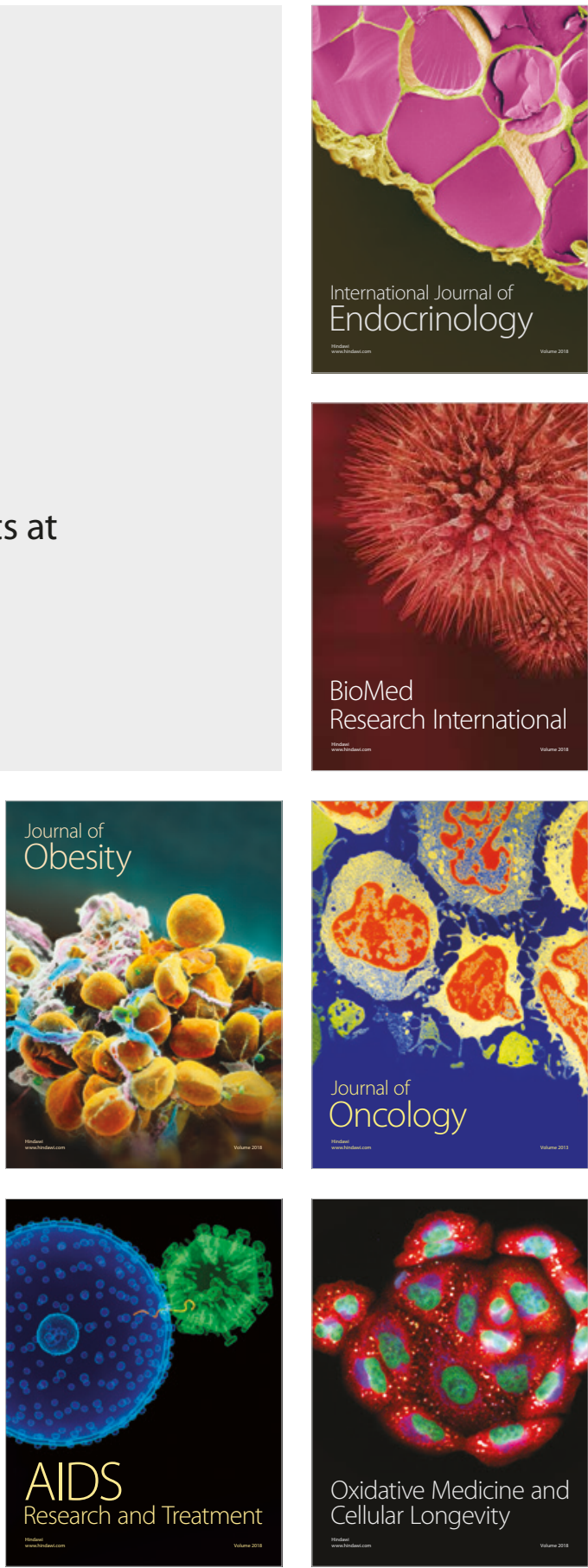